The ASTROPHySical Journal SuPPLEMENT SERIES, 90:917-922, 1994 February

C1994. The American Astronomical Society. All rights reserved. Printed in U.S.A.

\title{
HIGH-ENERGY GAMMA-RAY OBSERVATIONS OF ACTIVE GALAXIES
}

\author{
CARL E. Fichtel \\ NASA/Goddard Space Flight Center, Greenbelt, MD 20771 \\ Received 1993 February 22; accepted 1993 May 19
}

\begin{abstract}
During the period from 1992 May to early 1992 November, the Energetic Gamma-Ray Experiment Telescope (EGRET) on board the Compton Gamma Ray Observatory obtained high-energy gamma-ray data for most of the sky. A total of 18 active galaxies have been seen with high certainty, and it is expected that more will be found in the data when a more thorough analysis is complete. All of those that have been seen are radio-loud quasars or BL Lacertae objects; most have already been identified as blazars. No Seyfert galaxies have been found thus far. If the spectra are represented as a power law in energy, spectral slopes ranging from approximately -1.7 to -2.4 are found. A wide range of $z$-values exists in the observed sample, eight having values in excess of 1.0 . Time variations have been seen, with the timescale for a significant change being as short as days in at least one case. These results imply the existence of very large numbers of relativistic particles, probably close to the central object. Although a large extrapolation is required, their existence also suggests that these active galactic nuclei may be the source of the extragalactic cosmic rays.
\end{abstract}

Subject headings: acceleration of particles — galaxies: active — gamma rays: observations — quasars: general

\section{INTRODUCTION}

The significance of measurements of high-energy gamma radiation from active galactic nuclei (AGNs) has long been recognized because of direct relationship of high-energy gamma rays to relativistic charged particles. However, prior to the launch of the Compton Gamma Ray Observatory $(C G R O)$, only one active galaxy had been observed in highenergy ( $E>30 \mathrm{MeV}$ ) gamma rays, 3C 273, and it was just barely detectable (Swanenburg et al. 1978). Now the situation is quite different. During the period from 1991 April to 1992 November, CGRO conducted an all-sky survey, and already, with only a partial analysis of the Energetic Gamma Ray Experiment Telescope (EGRET) data, a large number of quasars have been seen and their properties measured.

In this paper, after a very brief description of the EGRET telescope and the observations, the results on AGNs will be summarized, with particular attention being given to those which appear to be relevant to the topic of this conference, namely, particle acceleration. This section will be followed by a discussion of the deductions from this new information that seem plausible for the relativistic particles that must be present and a consideration of the implications for the nature of the environment of these particles and the conditions that would be needed to accelerate them. Finally, the question will be addressed of whether these AGNs, which are apparently capable of the acceleration of large quantities of relativistic particles, could be the source of the highest energy cosmic rays.

\section{THE EGRET INSTRUMENT AND OBSERVATIONS}

EGRET, one of four instruments flown on CGRO, has the standard elements of a high-energy gamma-ray telescope, specifically an anticoincidence scintillator dome to discriminate against charged particles, a particle track detector consisting of spark chambers with interspersed high- $Z$ material to convert the gamma rays into electron pairs, a trigger telescope which detects the presence of the pair and determines that the particles have the correct direction of motion, and an energy measurement device, which in the case of EGRET is a $\mathrm{NaI}(\mathrm{TI})$ crystal. A description of the instrument and its general capabilities is given by Kanbach et al. (1988). The results of the instrument calibration, both before and after launch, are given by Thompson et al. (1993a). The telescope covers the energy range from about $20 \mathrm{MeV}$ to $30 \mathrm{GeV}$. The effective area is about $1.5 \times 10^{3} \mathrm{~cm}^{2}$ from 0.2 to $1.0 \mathrm{GeV}$, and lower outside this range. The instrument is designed to be free of internal background, and the calibration tests have verified that it is at least an order of magnitude below the extragalactic gamma radiation. Hence the only significant radiation besides the sources themselves is the diffuse Galactic and extragalactic radiation.

To ensure that the association of observed sources with AGNs was not contaminated by chance coincidences except at a very low level, an a priori list of radio-loud quasars and BL Lac objects was developed. "Radio-loud" was defined as having an intensity of approximately 1 jansky or more at about 1 $\mathrm{GHz}$. The list was found to contain 502 sources. Based on the typical positional uncertainties, there is less than a $1 \%$ chance of an accidental coincidence for a given source. The total number of certain sources not in the Galactic plane seen thus far is 24 , of which 18 are seen to be associated with objects in this list. The others are not identified with any obvious sources detected at other wavelengths. Hence, the detected radio quasar and BL Lacertae list is not likely to contain more than one chance coincidence, and more probably it will contain none.

\section{SUMMARY OF THE RESULTS}

The preliminary analysis of the EGRET data has revealed a total of 18 certain detections of radio-loud quasars and BL Lac objects. Table 1 summarizes these EGRET AGN detections. 
TABLE 1

Characteristics of Active Galaxies Detected by EGRET

\begin{tabular}{|c|c|c|c|c|c|c|c|c|c|c|c|c|}
\hline \multirow[b]{2}{*}{$\begin{array}{c}\text { SOURCE } \\
\text { IDENTIFICATION }\end{array}$} & \multicolumn{6}{|c|}{ SOURCE ChaRaCteristics } & \multirow[b]{2}{*}{$\begin{array}{c}\text { POSITION } \\
\text { DIFFERENCE }\end{array}$} & \multirow[b]{2}{*}{$\begin{array}{c}\text { POSITION } \\
\text { UNCERTAINTY }^{c}\end{array}$} & \multirow{2}{*}{$\begin{array}{c}\text { FLUX } \\
\left(10^{-6} \mathrm{~cm}^{-2} \mathrm{~s}^{-1}\right) \\
(E>100 \mathrm{MeV})\end{array}$} & \multirow{2}{*}{$\begin{array}{l}\text { PHOTON } \\
\text { SPECTRAL } \\
\text { INDEX }\end{array}$} & \multirow[b]{2}{*}{$z$} & \multirow[b]{2}{*}{$\begin{array}{l}\text { RELATIVE } \\
\text { LUMINOSITY }\end{array}$} \\
\hline & OVV & $\begin{array}{l}\mathrm{BL} \\
\mathrm{Lac}\end{array}$ & Superlumal & $\begin{array}{l}\text { Radio- } \\
\text { loud }\end{array}$ & $\begin{array}{c}\text { Flat } \\
\text { Radio }^{a}\end{array}$ & $\begin{array}{c}\text { Optical } \\
\text { Polarization }^{\mathrm{a}}\end{array}$ & & & & & & \\
\hline $0202+149(4 C+15.05) \ldots$ & & & & $\mathrm{x}$ & $\mathrm{x}$ & $\mathrm{x}$ & 0.3 & 0.4 & $0.3 \pm 0.1$ & & & \\
\hline PKS 0208-512 $\ldots \ldots \ldots$ & & & & $\mathrm{x}$ & $\mathrm{x}$ & $\mathrm{x}$ & 0.13 & 0.13 & $0.4-0.9$ & $-1.7 \pm 0.1$ & 1.00 & 2 \\
\hline $0235+164(\mathrm{OD}+160) \ldots \ldots$ & & $\mathrm{x}$ & $\mathrm{x}$ & $\mathrm{x}$ & $\mathrm{x}$ & $\mathrm{x}$ & 0.10 & 0.3 & $0.8 \pm 0.1$ & $-2.0 \pm 0.2$ & 0.94 & 2.0 \\
\hline 0420-014 (OA 129). & $\mathrm{x}$ & & & $\mathrm{x}$ & $\mathrm{x}$ & $\mathrm{x}$ & 0.5 & 0.4 & $0.4 \pm 0.1$ & $\ldots$ & 0.92 & 0.4 \\
\hline PKS 0454-463 $\ldots \ldots \ldots$ & & & & $\mathrm{x}$ & $\mathrm{x}$ & & 0.27 & 0.38 & $0.25 \pm 0.1$ & & 0.86 & 0.3 \\
\hline PKS $0528+134 \quad \ldots \ldots \ldots$ & $\mathrm{x}$ & & & $\mathrm{x}$ & $\mathrm{x}$ & & 0.13 & 0.15 & $0.4-1.6$ & $-2.4 \pm 0.1$ & 2.06 & $4-13$ \\
\hline PKS $0537-441 \quad \ldots \ldots \ldots$ & & $\mathrm{x}$ & & $\mathrm{x}$ & $\mathrm{x}$ & $\mathrm{x}$ & 0.4 & 0.6 & $0.3 \pm 0.1$ & $-2.0 \pm 0.2$ & 0.894 & 0.2 \\
\hline $0716+714 \ldots \ldots \ldots \ldots$ & & $\mathrm{x}$ & ? & $\mathrm{x}$ & $\mathrm{x}$ & & 0.47 & 0.4 & $0.20 \pm 0.06$ & $-1.8 \pm 0.2$ & $\ldots$ & \\
\hline $0836+710(4 \mathrm{C}+71.07) \ldots$ & $\mathrm{x}$ & & $\mathrm{x}$ & $\mathrm{x}$ & $\mathrm{x}$ & & 0.58 & 0.50 & $0.15 \pm 0.04$ & & 2.17 & 1.1 \\
\hline $1101+384($ Mrk 421) .... & & $\mathrm{x}$ & $\mathrm{x}$ & $\mathrm{x}$ & $\mathrm{x}$ & & 0.3 & 0.4 & $0.14 \pm 0.03$ & $-1.9 \pm 0.1$ & 0.031 & 0.0002 \\
\hline $1226+023(3 \mathrm{C} 273) \ldots \ldots$ & & & $\mathrm{x}$ & $\mathrm{x}$ & $\mathrm{x}$ & $\mathrm{x}$ & 0.2 & 0.5 & $0.30 \pm 0.05$ & $-2.4 \pm 0.1$ & 0.158 & 0.008 \\
\hline $1253-055(3 \mathrm{C} 279) \ldots \ldots$ & $\mathrm{x}$ & & $\mathrm{x}$ & $\mathrm{x}$ & $\mathrm{x}$ & $\mathrm{x}$ & 0.083 & 0.08 & $0.6-4.9$ & $-2.0 \pm 0.1$ & 0.54 & $0.3-2$ \\
\hline $1406-076 \ldots \ldots$ & & & & $\mathrm{x}$ & $\mathrm{x}$ & & 0.2 & 0.4 & $1.0 \pm 0.2$ & $\ldots$ & 1.49 & 2 \\
\hline $1606+106(4 C+10.45) \ldots$ & & & & $\mathrm{x}$ & $\mathrm{x}$ & & 0.42 & 0.50 & $0.5 \pm 0.2$ & $\ldots$ & 1.23 & 1.6 \\
\hline $1633+382(4 C+38.41) \ldots$ & $\mathrm{x}$ & & & $\mathrm{x}$ & $\mathrm{x}$ & & 0.08 & 0.15 & $0.4-1.4$ & $-2.0 \pm 0.1$ & 1.81 & $3-11$ \\
\hline $2052-474 \quad \ldots \ldots \ldots \ldots \ldots$ & & & & $\mathrm{x}$ & $\mathrm{x}$ & & 0.35 & 0.5 & $0.3 \pm 0.1$ & & 1.489 & 0.6 \\
\hline $2230+114($ CTA 102$) \ldots$ & & & $\mathrm{x}$ & $\mathrm{x}$ & $?$ & $\mathrm{x}$ & 0.3 & 0.4 & $0.24 \pm 0.07$ & $-2.4 \pm 0.1$ & 1.037 & 0.5 \\
\hline $2251+158(3 C 454.3) \ldots \ldots$ & $\mathrm{x}$ & & $\mathrm{x}$ & $\mathrm{x}$ & $\mathrm{x}$ & $\mathrm{x}$ & 0.25 & 0.22 & $0.8 \pm 0.1$ & $-2.0 \pm 0.1$ & 0.859 & 0.5 \\
\hline
\end{tabular}

Flat-spectrum radio sources: $\alpha_{r}>-0.5(2-5 \mathrm{GHz}$ band); optical polarization: polarization $>3 \%$.

${ }^{b}$ Difference between gamma-ray determined position and known position of identified source. Most are preliminary.

c There is a $68 \%$ probability that the source is within a circle of this radius. Most are preliminary.

d The source luminosity for the observed energy range $(0.1 \mathrm{GeV}<E<5 \mathrm{GeV})$, computed using the known redshift assuming $H_{0}=75 \mathrm{~km} \mathrm{~s}^{-1} \mathrm{Mpc}^{-1}$ and $q_{0}=\frac{1}{2}$, is equal to the relative luminosity times $\left(10^{48} \mathrm{ergs} \mathrm{s}^{-1}\right) f$, where $f$ is an unknown beaming factor. Typically $f$ is thought to be in the range from $10^{-2}$ to $10^{-3}$. 
The references for the individual detections may be found in Fichtel et al. (1993a), except for 2052-474, for which the reference is Thompson et al. (1993b), and 1406-076, for which the reference is Dingus et al. (1993). There are also several marginal detections, as would be expected, which need further study ( Thompson et al. 1993b). These are: 0454-234, $0521-365,0804+499,1510-089$, and $2356+196$. A very striking aspect of the results presented in this table is that nearly all of the observed AGNs are not the close ones; in fact, there is a broad distribution in $z$, with eight having values in excess of 1.0. Most are already known to be blazars. As noted earlier, these detections do not represent a complete or systematic survey, although the stronger sources at the time of the observation are probably in the list. Sources for which a range of fluxes is shown have exhibited significant variability. Note also that the spectra when represented by a power law in energy have spectral indices ranging from -1.7 to -2.4 . Two examples of spectra are shown in Figures 1 and 2.

Special attention is drawn to Figure 2. Mrk 421 (Lin et al. $1993 \mathrm{a}$ ) is the only one of the quasars and BL Lac objects seen by EGRET that has been seen at energies above the EGRET range, even though a search has been made for very high energy radiation from others. The measurements using the Whipple Observatory telescope (Punch et al. 1992) led to the intensity shown in Figure 2. This result shows that in at least one case the radiation extends not only to the several times $10^{9}-10^{10} \mathrm{eV}$ range but even to approximately $10^{12} \mathrm{eV}$.

As Table 1 shows, if the radiation were isotropic, typical

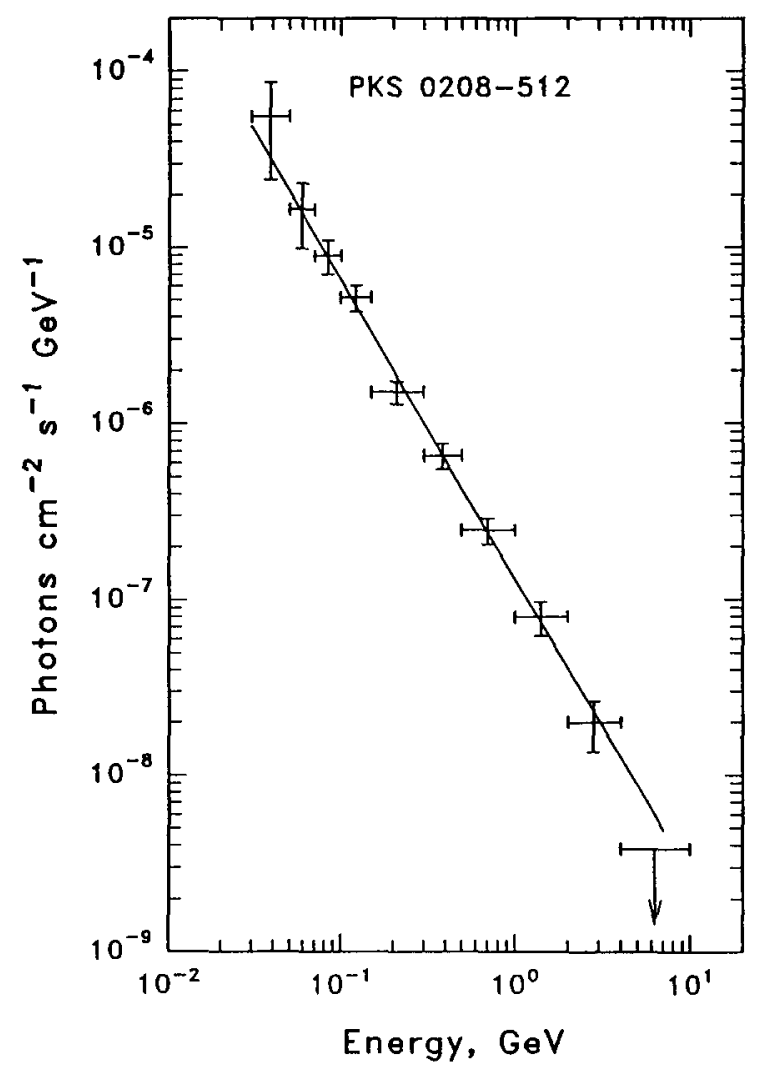

FIG. 1.-Differential energy spectrum of PKS 0208-512 seen by EGRET (Bertsch et al. 1993).

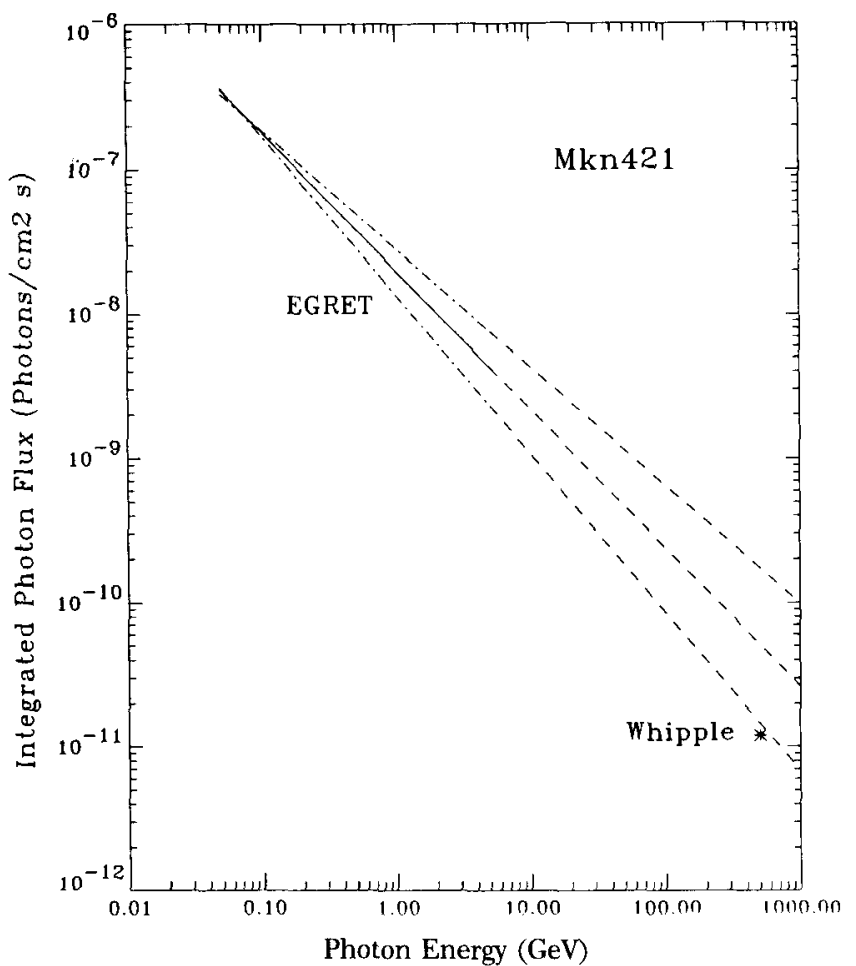

FIG. 2.-Differential energy spectrum of Mrk 421 seen by EGRET (Lin et al. 1993a), together with the very high energy observation of the Whipple Observatory (Punch et al. 1992).

values would be $10^{47}$ or $10^{48} \mathrm{ergs} \mathrm{s}^{-1}$. However, for reasons to be discussed later, it is more likely that they are beamed, probably with a beaming factor in the $10^{-2}$ to $10^{-3}$ range. In this case, the typical energy emission might be closer to $10^{-45}$ ergs $\mathrm{s}^{-1}$. Even this energy is extraordinary.

Several have been observed to be variable in time. In most instances, and there are several examples, the timescale is several weeks or months, i.e., from one observation to the next. However, in one case, the observation of 3C 279 from 1991 June 16 to 28 , the timescale of the variation was seen to be of the order of a day. The observed intensity as a function of time for this period is shown in Figure 3.

Another preliminary result of the EGRET all-sky survey that should be mentioned here, because of its relevance to the later discussion, is that no Seyfert galaxy has been observed in high-energy gamma rays ( Lin et al. 1993b). It is worth noting that the Oscillating Scintillation Spectrometer Experiment (OSSE) of $C G R O$ has already reported the positive detection of seven Seyferts and possible detection of two others in lowenergy gamma rays (Cameron et al. 1993); at the same time, only two quasars have been seen (3C 273 and 3C 279) at low energies by OSSE. A special search was made in the EGRET data for some of the Seyferts which were particularly intense at lower energies, including NGC 4151, Mrk 509, MCG +8-1111 , and NGC 1275, and the result was still null (Lin et al. $1993 b)$. Only upper limits in the range of $(0.5-1.2) \times 10^{-7}$ photons $\mathrm{cm}^{-2} \mathrm{~s}^{-1}$ were obtained. A rather considerable change in the spectral shape between the low- and high-energy gamma-ray results is in fact required to explain the observations for this last group. 


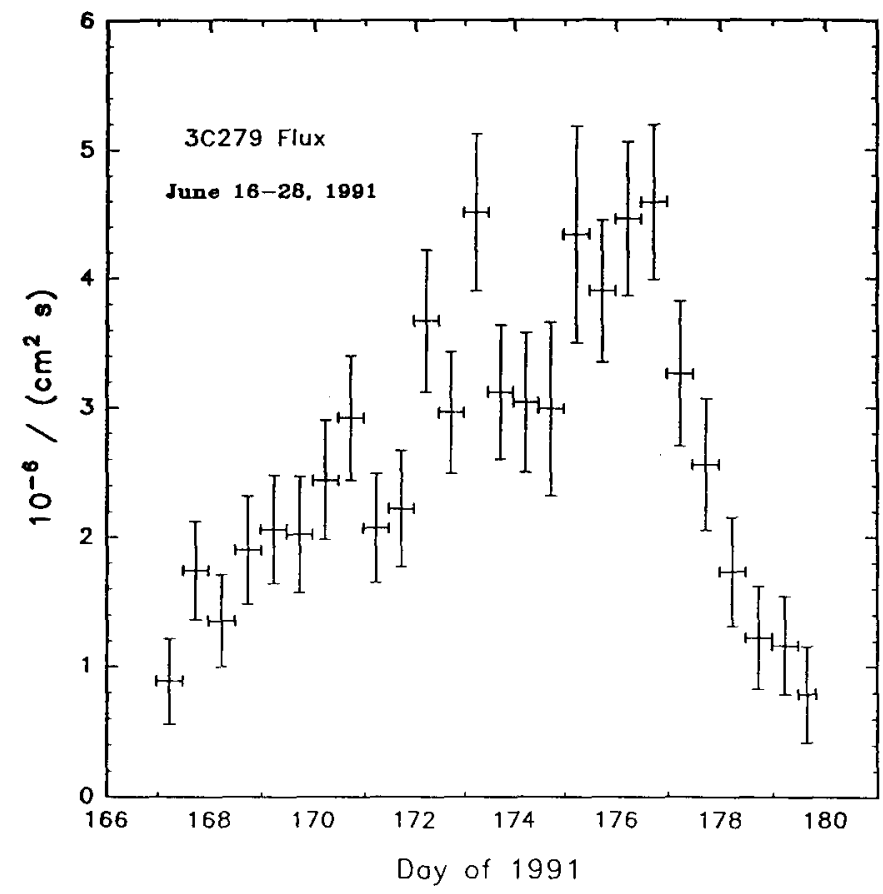

FIG. 3.-Flux of gamma rays from 3C 279 with energies above 100 MeV observed by EGRET for the 1991 June 16-28 period (Kniffen et al. 1993).

\section{DISCUSSION OF THE RESULTS AND IMPLICATIONS FOR PARTICLE ACCELERATION}

With the results that have just been presented, together with knowledge of these objects gained from observations, a number of inferences may be drawn about the objects in general and particle acceleration in particular. The two which are perhaps the most striking and immediately apparent are the total energy being emitted per second and the timescale for change for objects so distant and energy releases so large. As noted in $\S 3$, even assuming beaming, the energies being emitted in several cases are of the order of $10^{45} \mathrm{ergs} \mathrm{s}^{-1}$, yet several are already known to be variable in high-energy gamma-ray emission, with one having been seen to have a marked change in about 1 day.

For the moment, assume that there is a supermassive black hole at the center of a blazar. For a $10^{10} M_{\odot}$ black hole, $R_{\mathrm{S}}$, the Schwarzschild radius, is about 1 light-day. Time dilation must, of course, be taken into account. However, assuming that the observed variations are directly related to the size of the region, even with a somewhat smaller black hole and a significant time dilation which would be expected in a beam direction approximately toward the Earth, the observed change in 1 day seen for 3C 279 implies that the radiation is more probably coming from a region quite close to the supermassive black hole, and not, for example, at some great distance in the outer part of a jet, although the latter is possible, in principle, for a tightly spiraled jet.

There seem to be several factors which point to the radiation being associated with a jet. First is the correlation with objects which demonstrate jet characteristics at other wavelengths. In addition to all the observed AGNs being radio-loud and having flat spectra, most are either BL Lac objects or have already been observed to be optically violently variable (OVV), superluminal, or optically polarized. Second, the wide distribution in $z$ suggests that there is something unusual; not all quasars, nor even radio-loud ones, emit high-energy gamma rays at this level, at least not in the direction of the Earth, or the sky would be seen in high-energy gamma rays at an intensity many orders of magnitude above what is observed. The hard spectra to high energies and time variations are more consistent with beam models than with isotropic emission models. In fact, it is rather hard to conceive of an isotropic model in which the amount of energy emitted and the time variation are consistent with observations.

If one accepts this reasoning, the high-energy gamma-ray results can then be taken as support for the similar nature of OVV and other beamed quasars and BL Lac objects, i.e., blazars. Further, although not providing absolute proof, the observation of this very large amount of energy coming from a region of only about $10^{16} \mathrm{~cm}$ or a bit more adds support to at least blazars being supermassive black holes. What else could they be?

A unified picture of AGNs based on the theoretical idea of an accretion disk, surrounded by a thick torus of cooler gas, feeding a massive black hole, sometimes with a jet of particles emerging along the axis of the disk, had already emerged before the EGRET observations discussed here. This picture is supported by the observation of radio-frequency jets, superluminal motion in some sources, and other observations (for a review see Osterbrock 1991). A basic premise of this picture is that all AGNs are different aspects of the same phenomenon. Different classes of AGNs look different primarily because they are viewed at different angles on the sky. The hypothesized relationships are as follows: in the case of OVV quasars the jet is aimed almost directly at the observer, while in Seyfert 1 and 2 galaxies the line of sight is oriented at a substantial angle with respect to the axis of the disk and torus. BL Lac objects are proposed to be extreme examples of the quasar population with the jets seen almost exactly end-on (Blandford \& Rees 1978; Blandford \& Königl 1979). The detection of high-energy gamma emission from blazars and its nondetection from Seyfert galaxies give further support to this picture. The situation is probably more complicated than that just described, particularly in regard to BL Lac objects (see, e.g., Valtaoja et al. 1992; Impey 1992; Fichtel et al. 1993b), but this picture may have some merit for general considerations.

\subsection{Origin of the High-Energy Gamma Rays}

Assuming that a relativistic jet exists, it is very difficult to conceive of a method of producing high-energy gamma rays in such large intensities with the observed time variations except with the existence of a beam of relativistic particles. Within the framework of a beam of relativistic particles, however, there are a variety of different models leading to the emission of high-energy gamma rays. They can be broadly classified as either models involving Compton scattering (Ghisellini, Maraschi, \& Treves 1985; Jones, O’Dell, \& Stein 1974; Gould 1979; Maraschi, Ghisellini, \& Celotti 1992) or nucleonic cascade models (Kazanas \& Ellison 1986; Marscher \& Bloom 1992). In the Compton scattering models, gamma-ray emis- 
sion could arise either via the synchrotron-self-Compton process or from Compton scattering of UV flux from an accretion disk. The nucleonic cascade would involve both bremsstrahlung and nucleon-nucleon interactions leading to a combined spectrum similar to that of the diffuse Galactic gamma radiation, and hence similar to that observed for the AGNs described above.

\subsection{Particle Acceleration}

There are then at least two further questions to be addressed, namely, the source of energy and its conversion to relativistic particles. Beginning with the first, there seems to be general agreement that the basic energy supply is provided by matter falling into the very deep gravitational potential well around a supermassive black hole. There seems in fact to be little difficulty in accounting for the overall energy of an AGN in this manner. How does an adequate fraction of this energy become converted to relativistic particle energy, and a portion of that to extreme relativistic particle energy? If this approach is to be successful, a reasonable model for the natural evolution of a large, compact ( recall the time variation ), reasonably uniform magnetic field must be found.

Consider an analogy to a familiar model, the case of isolated pulsars; it seems clear that the dominant role in producing acceleration of charged particles is played by a rotating magnetic field configuration. Extending this conclusion to AGNs is not trivial. The formation of a uniform magnetic field is not an immediate natural consequence of the origin of a supermassive black hole, as it is in the case of a neutron star. Hence, if this approach is to be successful, a reasonable model for the natural evolution of a large, compact, and reasonably uniform magnetic field must be found. The Blandford-Znajek (1977) process is a plausible one. In this model, a conducting accretion disk around a massive rotating black hole serves to confine a magnetic field that threads the hole's horizon. The horizon acts as a rotating conductor immersed in an external magnetic field, i.e., a unipolar generator that can deliver power to a magnetospheric load. Apparently, if energy can be extracted in this way, then relativistic beams emerge along the rotation axis of the hole in a natural way. This power generation process is very similar to a process originally proposed for neutron stars by Goldreich \& Julian (1969). A particularly illuminating analysis of the Blandford-Znajek process is given by MacDonald \& Thorne (1982).

The key question is the manner in which the intense uniform magnetic field threading the black hole becomes established in the first place. No one known to this author has succeeded in proving with mathematical rigor that such a configuration is an inevitable result of the physical situation. However, MacDonald et al. (1986) have provided qualitative reasoning based on the natural evolution of the magnetic field near the large black hole from a combination of Maxwell pressure and Maxwell tension considerations. They further argue that no matter how chaotic the field threading the disk may be, the field through the hole will be very clean and ordered.

The potential drop available for particle acceleration is approximately

$$
\Delta V=\left(10^{20} \mathrm{~V}\right)\left(a / M_{\mathrm{BH}}\right)\left(B / 10^{4} \mathrm{G}\right)\left(M_{\mathrm{BH}} / 10^{9} M_{\odot}\right),
$$

where $B$ is the magnetic field threading the horizon, $M_{\mathrm{BH}}$ is the mass of the black hole, and $a$ is the angular momentum per unit mass of the black hole. For reasonable parameters, as much as $10^{20}-10^{21} \mathrm{~V}$ is available. If the charged particle existed in a vacuum, then it could be accelerated up to a maximum energy of $e \Delta V$. But many effects can limit the energy to much lower values. These include synchrotron radiation losses and Compton cooling. In a plasma, collisions with other particles can limit the maximum attainable energy. Even if the plasma is tenuous and collisions are unimportant, a variety of plasma microinstabilities may also limit the energy. Various stochastic reacceleration mechanisms, such as Fermi shock acceleration, may act to overcome some of these losses.

The total power in the relativistic beams is of order

$$
\begin{aligned}
L_{b}=\left(10^{45} \operatorname{ergs~s}^{-1}\right)( & \left(a / M_{\mathrm{BH}}\right)^{2} \\
& \times\left(B / 10^{4} \mathrm{G}\right)^{2}\left(M_{\mathrm{BH}} / 10^{9} M_{\odot}\right)^{2} .
\end{aligned}
$$

For reasonable astrophysical parameters the beam luminosity can approach $10^{45}-10^{47}$ ergs s $^{-1}$, consistent with observations.

Lovelace (1976) proposed a different model, in which magnetic fields generated in a differentially rotating disk can lead to acceleration of charged particles to ultrarelativistic energies and extraction of energy from the disk. The model apparently can produce either a proton beam or an electron-positron beam, depending on whether $B_{z}$ is parallel or antiparallel to the angular momentum vector of the disk. The potential drop available for particle acceleration and the beam luminosity in this case are comparable to the above estimates for the Blandford-Znajek effect.

\subsection{Implication for Extragalactic Cosmic Rays}

As noted above, the intensity and spectral slopes of most of the observed quasars and BL Lac objects suggest the presence of an extraordinary source of relativistic particles with spectra even slightly harder than the observed ultra-high-energy cosmic rays. The beaming into a very narrow cone combined with the knowledge gained from the study of high-energy nuclear physics interactions suggests that the parent particles are extremely relativistic. Finally, one of the quasars already seen by EGRET and discussed here, namely, Mrk 421, has already been detected in gamma rays at $10^{12} \mathrm{eV}$.

All of these considerations bring to mind the possibility suggested by Colgate (1990), among others, that blazar jets may be the source of the ultra-high-energy cosmic rays, those above about $10^{18} \mathrm{eV}$. There is, of course, a very long step from a few times $10^{9} \mathrm{eV}$ or even $10^{12} \mathrm{eV}$ gamma rays to $10^{18}-10^{21} \mathrm{eV}$ cosmic rays, but the acceleration mechanism and the energy could be there for a supermassive black hole at the center of an AGN, as discussed above. However, the answers to very critical questions are all at the moment unknown. For example: Are the particles actually accelerated to $10^{18}-10^{21} \mathrm{eV}$ ? Do they escape from the AGN? What is their energy spectrum?

\section{SUMMARY}

At the present time, data from the EGRET instrument have led to the detection in high-energy gamma-rays of 18 radioloud, flat-spectrum quasars and BL Lac objects, most of which 
have already been established as blazars, as well as the marginal detection of others which need further confirmation. No Seyfert galaxy has been seen. Several of the detected AGNs are seen to be time-variable, one on the order of days. If the spectra are represented as power laws, energy spectra in the range -1.7 to -2.4 are found. The amount of energy released, the association with blazars, and other considerations suggest that the origin of the high-energy gamma rays is a jet of a very large number of relativistic charged particles probably interacting near the central object of the AGN, presumably a supermassive black hole. The possibility that these objects are the source of the very high energy extragalactic cosmic rays seems now to have new support.

\section{REFERENCES}

Bertsch, D. L., et al. 1993, ApJ, 405, L21

Blandford, R. D., \& Königl, A. 1979, ApJ, 232, 34

Blandford, R. D., \& Rees, M. J. 1978, in Pittsburgh Conf. on BL Lac Objects, ed. A. M. Wolfe (Pittsburgh: Univ. Pittsburgh Press), 328

Blandford, R. D., \& Znajek, R. 1977, MNRAS, 179, 433

Bloom, S., \& Marscher, A. 1992, in Proc. Compton Observatory Science Workshop ( NASA CP-3137), 578

Cameron, R. A., et al. 1993, in AIP Conf. Proc., Compton Gamma Ray Observatory, ed. M. Friedlander, N. Gehrels, \& D. J. MacComb (New York: AIP), 478

Colgate, S. 1990, paper presented at AAS Meeting, 1990 June 10-14, paper 51.01

Dingus, B. L., et al. 1993, IAU Circ., No. 5690

Fichtel, C. E., et al. 1993a, in AIP Conf. Proc., Compton Gamma Ray Observatory, ed. M. Friedlander, N. Gehrels, \& D. J. MacComb (New York: AIP), 1194

. 1993b, in AIP Conf. Proc., Compton Gamma Ray Observatory, ed. M. Friedlander, N. Gehrels, \& D. J. MacComb (New York: AIP), 461

Ghisellini, G., Maraschi, L., \& Treves, A. 1985, A\&A, 146, 204

Goldreich, P., \& Julian, W. H. 1969, ApJ, 157, 869

Gould, R. J. 1979, A\&A, 76, 306

Impey, C. D. 1992, in Variability of Blazars, ed. E. Valtaoja \& M. Valtonen (Cambridge: Cambridge Univ. Press), 55
Jones, T. W., O'Dell, S. L., \& Stein, W. A. 1974, ApJ, 188, 353

Kanbach, G., et al. 1988, Space Sci. Rev., 46, 69

Kazanas, D., \& Ellison, D. C. 1986, Nature, 319, 380

Kniffen, D. A., et al. 1993, ApJ, 411,133

Lin, Y. C., et al. 1993a, ApJ, 416, L53 1993b, ApJ, submitted

Lovelace, R. V. E. 1976, Nature, 262,649

MacDonald, D., \& Thorne, K. S. 1982, MNRAS, 198, 345

MacDonald, D. A., Thorne, K. S., Price, R. H., \& Zhang, X-H. 1986, in Black Holes: The Membrane Paradigm, ed. K. P. S. Thorne, Richard H. Price, \& Douglas A. MacDonald (New Haven: Yale Univ. Press), chap. 4

Maraschi, L., Ghisellini, G., \& Celotti, A. 1992, in AIP Conf. Proc. 254, Testing the AGN Paradigm, ed., S. Holt, S. G. Neff, \& C. M. Urry (New York: AIP), 439

Osterbrock, D. E. 1991, Rep. Progr. Phys., 54, 579

Punch, M., et al. 1992, Nature, 358, 477

Swanenburg, B. N., et al. 1978, Nature, 275, 298

Thompson, D. J., et al. 1993a, ApJS, 86, 629 1993b, BAAS, Vol. 24, paper 21.04

Valtaoja, E., Terasranta, H., Lainela, M., \& Teerikorpi, P. 1992, in Variability of Blazars, ed. E. Valtaoja \& M. Valtonen (Cambridge: Cambridge Univ. Press), 70 